of $N$ which satisfy this condition, are found by a trial and error process from Eqs. (8) and (9). It is interesting to note that Eq. (9), for $x= \pm a$, is divergent for all values of $N$ except those corresponding to the modes of vibration satisfying the second boundary condition.

In Fig. $1, X / A_{1}$, has been plotted as a function of $x / a$ for various values of $N$, the thickly-drawn curves representing the "standing-wave" patterns for the fundamental mode and its first two overtones. It was sufficient to show the deflection variations over only one half the length of the ellipsoid because there is symmetry about the plane $x=0$.

The frequencies of resonance for the ellipsoid may thus be expressed as

$$
f_{R_{n}}=\frac{n}{4 a}\left(\frac{k}{\rho}\right)^{1 / 2} \quad \text { where } n \simeq 1.38,3.49,5.33, \cdots,
$$

indicating the inharmonic nature of the overtones. However, a comparison between Eqs. (3) and (10) reveals that the consecutive overtones of the vibrating ellipsoid have ratios closer and closer to the ratios of adjacent odd integers as the value of $n$ increases.

Acknowledgements. I wish to thank Professor L. W. McKeehan of this laboratory, whom I am assisting in the study of magnetostrictive vibration, and Professor F. J. Beck, Jr., of the Dunham Laboratory of Electrical Engineering, for encouraging discussions of this problem.

\title{
THE LEAST SQUARES SOLUTION FOR A SET OF COMPLEX LINEAR EQUATIONS*
}

By R. TURETSKY (Aberdeen Proving Ground)

Consider the set of $m$ observational equations whose matrix representation is

$$
A z \sim w,
$$

where $A$ is an $m \times n(m \geq n)$ matrix of rank $n$ whose elements are prescribed complex quantities, while $w$ is an $m \times 1$ matrix. We seek that $z$ (an $n \times 1$ matrix) which minimizes the sum of the squares of the absolute values of the components of the vector $A z-w$.

Set $A=B+i C, z=x+i y, w=u+i v$, where $B, C, x, y, u$, and $v$ are real matrices. Then Eq. (1) is equivalent to

$$
\left(\begin{array}{rr}
B & -C \\
C & B
\end{array}\right)\left(\begin{array}{l}
x \\
y
\end{array}\right) \sim\left(\begin{array}{l}
u \\
v
\end{array}\right)
$$

This is the matrix representation for a set of real observational equations. To obtain the normal equations, ${ }^{1}$ we multiply on the left by the transpose of the coefficient matrix

${ }^{*}$ Received July 28, 1950.

'See Whittaker and Robinson, Calculus of observations, Chapter IX. 
of the unknowns. Letting primes denote the transpose, there results

$$
\left(\begin{array}{rr}
P & -Q \\
Q & P
\end{array}\right)\left(\begin{array}{l}
x \\
y
\end{array}\right)=\left(\begin{array}{cc}
B^{\prime} & C^{\prime} \\
-C^{\prime} & B^{\prime}
\end{array}\right)\left(\begin{array}{l}
u \\
v
\end{array}\right),
$$

where

and

$$
P=B^{\prime} B+C^{\prime} C
$$

$$
Q=C^{\prime} B-B^{\prime} C
$$

It will be noted that $P$ is svmmetric while $Q$ is skew-symmetric, which greatly facilitates the computation.

The solution of Eq. (3) is

$$
\left(\begin{array}{l}
x \\
y
\end{array}\right)=\left(\begin{array}{rr}
P & -Q \\
Q & P
\end{array}\right)^{-1}\left(\begin{array}{ll}
B^{\prime} & C^{\prime} \\
C^{\prime} & B^{\prime}
\end{array}\right)\left(\begin{array}{l}
u \\
v
\end{array}\right)
$$

Ordinarily the inverse of the $2 n \times 2 n$ matrix

$$
\left(\begin{array}{rr}
P & -Q \\
Q & P
\end{array}\right)
$$

is obtained directly. However, recourse may be had to its special structure to note that the inverse is of the form

$$
\left(\begin{array}{rr}
R & S \\
-S & R
\end{array}\right)
$$

where

$$
\begin{aligned}
& R=\left(P+Q P^{-1} Q\right)^{-1} \\
& S=P^{-1} Q R
\end{aligned}
$$

The computation is thus simplified, since we have to compute the inverses of two $n \times n$ matrices rather than that of one $2 n \times 2 n$ matrix. Moreover, advantage can be taken of the fact that $R$ is symmetric while $S$ is skew-symmetric.

Finally, the multiplication of

$$
\left(\begin{array}{rr}
R & S \\
-S & R
\end{array}\right)
$$

by

$$
\left(\begin{array}{cc}
B^{\prime} & C^{\prime} \\
-C^{\prime} & B^{\prime}
\end{array}\right)
$$


required on the right hand side of Eq. (5) leads to the matrix

$$
\left(\begin{array}{rr}
T & -U \\
U & T
\end{array}\right)
$$

where

$$
\begin{aligned}
& T=R B^{\prime}-s C^{\prime} \\
& U=R C^{\prime}+s B^{\prime}
\end{aligned}
$$

Multiplication by a $2 m \times 2 n$ matrix is thus required only in the final step. 